\title{
Michel ROCHE
}

Politologue, Université du Québec à Chicoutimi et chercheur associé à la chaire Hector-Fabre, UQAM

(2008)

\section{"La question nationale au Québec et l'éclatement des fédérations soviétique, tchécoslovaque et yougoslave: les limites de la comparaison.”}

\author{
Deuxième et dernier article.
}

Un document produit en version numérique par Jean-Marie Tremblay, bénévole, professeur de sociologie au Cégep de Chicoutimi

Courriel: jean-marie tremblay@uqac.ca

Site web pédagogique : http://www.uqac.ca/jmt-sociologue/

Dans le cadre de la collection: "Les classiques des sciences sociales"

Site web: http://classiques.uqac.ca/

Une collection développée en collaboration avec la Bibliothèque

Paul-Émile-Boulet de l'Université du Québec à Chicoutimi

Site web: http://bibliotheque.uqac.ca/ 


\section{Politique d'utilisation de la bibliothèque des Classiques}

Toute reproduction et rediffusion de nos fichiers est interdite, même avec la mention de leur provenance, sans l'autorisation formelle, écrite, du fondateur des Classiques des sciences sociales, Jean-Marie Tremblay, sociologue.

Les fichiers des Classiques des sciences sociales ne peuvent sans autorisation formelle:

- être hébergés (en fichier ou page web, en totalité ou en partie) sur un serveur autre que celui des Classiques.

- servir de base de travail à un autre fichier modifié ensuite par tout autre moyen (couleur, police, mise en page, extraits, support, etc...),

Les fichiers (.html, .doc, .pdf, .rtf, .jpg, .gif) disponibles sur le site Les Classiques des sciences sociales sont la propriété des Classiques des sciences sociales, un organisme à but non lucratif composé exclusivement de bénévoles.

Ils sont disponibles pour une utilisation intellectuelle et personnelle et, en aucun cas, commerciale. Toute utilisation à des fins commerciales des fichiers sur ce site est strictement interdite et toute rediffusion est également strictement interdite.

L'accès à notre travail est libre et gratuit à tous les utilisateurs. C'est notre mission.

Jean-Marie Tremblay, sociologue

Fondateur et Président-directeur général, LES CLASSIQUES DES SCIENCES SOCIALES. 
Cette édition électronique a été réalisée par Jean-Marie Tremblay, bénévole, professeur de sociologie au Cégep de Chicoutimi à partir de :

Michel Roche

Politologue, Université du Québec à Chicoutimi et chercheur associé à la chaire Hector-Fabre, UQAM

\section{"La question nationale au Québec et l'éclatement des fédéra- tions soviétique, tchécoslovaque et yougoslave: les limites de la comparaison."}

Un article publié dans la revue Bulletin d'histoire politique, vol. 16, no 3, printemps 2008, pp. 133-160. Montréal : Éditions Lux.

[Autorisation formelle accordée par l'auteur le 29 mars 2012 de diffuser cet article dans Les Classiques des sciences sociales.]

Courriel : Michel_Roche@uqac.ca

Polices de caractères utilisée :

Pour le texte: Times New Roman, 14 points.

Pour les citations : Times New Roman, 12 points.

Pour les notes de bas de page : Times New Roman, 12 points.

Édition électronique réalisée avec le traitement de textes Microsoft Word 2004 pour Macintosh.

Mise en page sur papier format : LETTRE US letter), 8.5'’ $\mathrm{x} 11^{\prime}$ '

Édition numérique réalisée le 20 mars 2012 à Chicoutimi, Ville de Saguenay, province de Québec, Canada. 
Cet article est la suite d’un précédent article :

Michel Roche

"L'éclatement des fédérations soviétique, yougoslave et tchécoslovaque dans le débat sur la question nationale au Québec : de l'échec de Meech au référendum de 1995."

Un article publié dans la revue Bulletin d'histoire politique, vol. 4, no 2, printemps 2007, pp. 151-176. Montréal : Éditions Lux.

http://dx.doi.org/doi:10.1522/030076183 


\section{Michel Roche}

Politologue, Université du Québec à Chicoutimi

et chercheur associé à la chaire Hector-Fabre, UQAM

\section{"La question nationale au Québec et l'éclatement des fédérations soviétique, tchécoslovaque et yougoslave : les limites de la comparaison."}

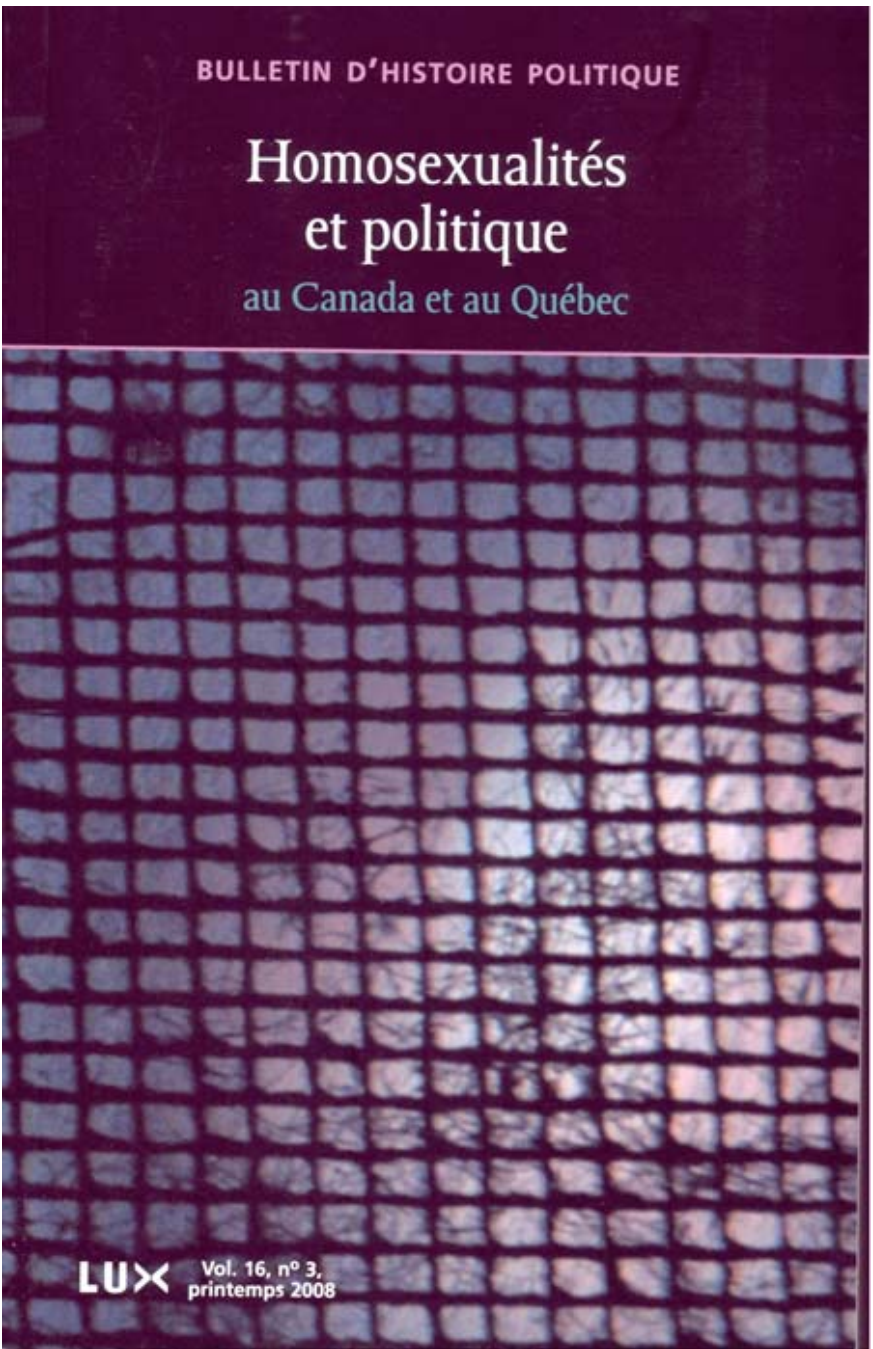

Un article publié dans la revue Bulletin d'histoire politique, vol. 16, no 3, printemps 2008, pp. 133-160. Montréal : Éditions Lux. 


\section{Table des matières}

Introduction

L’origine des fédérations de l'Est

La Yougoslavie

La Tchécoslovaquie

L'URSS

$\underline{\text { Les facteurs communs }}$

$\underline{\text { L’impact de la transition économique et de la démocratisation }}$

$\underline{\text { Conclusion }}$ 


\section{Michel Roche 1}

Politologue, Université du Québec à Chicoutimi

et chercheur associé à la chaire Hector-Fabre, UQAM

\section{"La question nationale au Québec et l'éclatement des fédérations soviétique, tchécoslovaque et yougoslave : les limites de la comparaison."}

Un article publié dans la revue Bulletin d'histoire politique, vol. 16, no 3, printemps 2008, pp. 133-160. Montréal : Éditions Lux.

\section{INTRODUCTION}

\section{$\underline{\text { Retour à la table des matières }}$}

L'éclatement de l'URSS, de la Tchécoslovaquie et de la Yougoslavie a eu un certain impact sur les débats qui ont précédé le référendum québécois du 30 octobre $1995^{2}$. Les deux camps y ont puisé les arguments qui appuyaient leurs thèses. Certains y ont vu la confirmation de leurs analyses au sujet d'un certain sens de l'histoire qui s'exprimerait par l'accession à l'indépendance de toutes les «nations». Le protonationalisme mènerait forcément à l’État-nation. "Ça s'applique aux pays qui ont quitté dernièrement l'Union soviétique comme ça s’applique à la République tchèque et à la République slovaque, et comme ça va s’appliquer au Québec bientôt » ${ }^{3}$. D’autres,

1 L'auteur est professeur de science politique à l’Université du Québec à Chicoutimi.

2 Voir Michel Roche, «L’éclatement des fédérations soviétique, yougoslave et tchécoslovaque dans le débat sur la question nationale au Québec : de l'échec de Meech au référendum de 1995», Bulletin d'histoire politique, Vol. 14, no. 3, printemps 2006, p. 171-192.

3 Intervention de Bernard Landry à l'Assemblée nationale, le 12 septembre 1995. Journal des débats, $35^{\mathrm{e}}$ législature, $1^{\text {ère }}$ session. 
cherchant à contrecarrer ce discours, ont fait valoir que l'éclatement de ces «fédérations» venait du caractère forcé de l'union des peuples concernés dans le cadre de régimes politiques dictatoriaux.

Trop souvent, les « lunettes » québécoises se limitent à l'aspect identitaire, c'est-à-dire aux différences linguistiques et culturelles et négligent par le fait même une série de facteurs absents ou qui se présentent sous une forme foncièrement différente dans le conflit Québec/Canada. D'où la conclusion selon laquelle le passage à la démocratie mènerait forcément à l'éclatement des fédérations de l'Est ${ }^{4}$. Il est vrai que la répression de toute velléité nationaliste en URSS ou en Yougoslavie constituait une caractéristique importante des régimes politiques en vigueur dans ces pays. Mais une telle vision des choses ne serait-elle pas trop réductrice? L'identité et l'altérité suffisentelles, en soi, à engendrer un mouvement revendiquant l’indépendance ?

Les concepts de nation, de nationalisme, d'identité nationale permettent de comprendre une certaine réalité. Il importe toutefois de ne jamais perdre de vue qu'il s'agit toujours de construits sociaux, de produits de l'histoire, qui ne sont pas donnés pour l'éternité. Les nations française, italienne ou allemande sont, comme toutes les autres, des constructions historiques. Il en va de même pour le Québec et pour toutes les communautés qui composaient l'URSS, la Yougoslavie et la Tchécoslovaquie. Des nations se sont érigées aux XVIIIe et XIXe siècles à partir de populations disparates parlant des dizaines de langues vernaculaires. Comme l'explique Anatoli Vichnevski,

« les différences culturo-linguistiques entre Russes, Ukrainiens et Biélorusses, entre Serbes et Croates, entre Tchèques et Slovaques ne sont pas plus importantes qu'entre, par exemple, Bretons, Provençaux et Alsaciens ; ils n’ont jamais connu autant de différends religieux aigus, et en

4 «Les contraintes de l’économie de marché ou celles plus étouffantes de l’internationalisme prolétarien n’ont pas réussi à extirper la volonté d’indépendance des peuples. Ainsi, en Union soviétique et en Yougoslavie, le désir d'indépendance des peuples subjugués a fait éclater ces deux fédérations pour donner naissance à de nouveaux pays indépendants». Denis Monière, «Les débats idéologiques», L'année politique au Québec 1990-1991, Québec/Amérique, 1992. 
tout cas pas plus que ceux qui ont divisé catholiques et protestants français ; ils étaient liés par un destin historique commun millénaire ${ }^{5}$ ».

Par ailleurs, la perception de constituer un peuple ou une communauté nationale ne se traduit pas forcément par la volonté d'accéder au statut d’État souverain. Des raisons économiques et sécuritaires peuvent également mener à la constitution d'États binationaux ou multinationaux.

En somme, l'éclatement des trois fédérations de l'Est ne peut s'expliquer de façon satisfaisante à partir des seuls critères identitaires. Dans les années qui ont précédé leur éclatement, les trois fédérations de l'Est s'étaient engagées dans une période de transition entre un régime fondé sur la propriété étatique des moyens de production et la planification centralisée et un autre caractérisé par la prédominance des rapports marchands et, éventuellement, des rapports capitalistes. Il s'agit là d'une distinction qui, à elle seule, met à l'épreuve toute comparaison avec le cas Québec/Canada.

Le but de cet article consiste donc à mettre en exergue les principaux facteurs ayant favorisé le démantèlement des fédérations de l'Est en insistant sur le caractère particulier du contexte dans lequel cela s'est produit. Sans nier les quelques similitudes avec le cas Québec/Canada, l'analyse mettra l'emphase sur les distinctions, trop souvent ignorées. L'analyse des causes de l'éclatement des fédérations de l'Est peut en outre faire œuvre utile dans le contexte où, au Québec, une aile du mouvement indépendantiste s'interroge sur la pertinence d'axer la stratégie d'accession à la souveraineté sur le retour au «nous» identitaire alors qu'une autre insiste toujours sur l'importance d'associer la lutte nationale du peuple québécois à un projet social fondé sur les revendications des salariés et des groupes communautaires.

5 Anatoli Vichnevski, « Des populations aux destins démographiques contrastés » in Dominique Colas (dir.), L'Europe post-communiste, Paris, PUF, 2002, p. 398. 


\title{
L’origine des fédérations de l'Est
}

\author{
La Yougoslavie
}

$\underline{\text { Retour à la table des matières }}$

La Yougoslavie a souvent été perçue comme le produit d'une union forcée de peuples aux griefs historiques insurmontables et maintenus au sein d'un même État d'abord par la poigne de fer de la dictature de la dynastie serbe avant la Deuxième Guerre mondiale, puis de la dictature de Tito et de la Ligue des communistes à partir de 1945. Ce point de vue laisse entendre qu'avec le déclin du régime communiste, l'indépendance devenait l'unique aboutissement possible. Une telle perception relève d'une méconnaissance de l'histoire. Le Royaume des Serbes, des Croates et des Slovènes, qui a pris le nom de Yougoslavie en 1929, émerge avec l'effondrement de l'empire austro-hongrois. Les origines idéologiques et politiques de cette union plongent au début du XIXe siècle, époque où le yougoslavisme, mouvement voué à la promotion de l'union des Slaves du sud, portait le nom d' «illyrisme ${ }^{6}$ ». Au-delà de la langue commune des Serbes et des Croates - les deux plus importantes nations du nouveau pays - des raisons de sécurité militaient en faveur de la création d'une entité plus large. On cherchait en effet à se protéger contre toute éventualité de prétention territoriale de la part de l'Allemagne, de l'Autriche, de la Hongrie et de l'Italie.

En dépit de la légitimité sur laquelle l'union reposait à ses tout débuts, la première Yougoslavie a été un échec, à la fois pour des raisons économiques et politiques. Le pays se situait à la périphérie de l'Europe et dépendait fortement des capitaux étrangers. Les inégalités régionales étaient particulièrement aiguës, les régions qui faisaient autrefois partie de l'Empire ottoman - Bosnie-Herzégovine, Kosovo, Monténégro, Macédoine - affichant un niveau de sous-développement qui tranchait nettement avec celui des autres. Le pays subissait en ou-

6 Jean-Arnault Dérens et Catherine Samary, Les conflits yougoslaves de A à Z, Éditions de l'Atelier et Éditions Ouvrières, Paris, 2000, p. 150-151. 
tre une dictature exercée par la dynastie serbe. Ces différents problèmes menèrent à l'éclatement de la Yougoslavie au début de la Seconde Guerre mondiale.

Toutefois, en dépit de la collaboration du régime croate des Oustachis avec l'Allemagne hitlérienne et des massacres de Serbes commis par ce régime, la résistance contre les fascistes trouvera des appuis chez tous les peuples de la Yougoslavie d'avant-guerre et formera la base d'une nouvelle union. La dictature titiste ne constituait pas la seule source d'unité : dans le contexte de la victoire sur l'Allemagne et l'Italie, victoire arrachée par une résistance déterminée, par des citoyens rompus à toutes les formes de combats et au prix de sacrifices immenses, il aurait été particulièrement difficile pour les communistes, relativement peu nombreux, de maintenir unis des peuples qui ne l'auraient pas souhaité ou, a fortiori, qui se seraient détestés comme cela semblait être le cas au début des années 1990 (les Serbes auraient très bien pu faire valoir leurs griefs contre les Croates, et ces derniers les leurs contre les Tchetniks serbes). Comme le rappelle Catherine Samary, « les différences culturelles, religieuses et historiques étaient perçues comme secondaires au regard de ce qui rapprochait 7 ".

La deuxième Yougoslavie a donc été fondée sur des intérêts communs relatifs à la sécurité, à l'économie et à la nécessaire solidarité sociale, condition absolument nécessaire pour rapprocher des communautés nationales disséminées sur un territoire ethniquement mélangé ${ }^{8}$. Le conflit politique et idéologique avec l'URSS de Staline suc-

7 Voir Catherine Samary, La déchirure yougoslave, Paris, L’Harmattan, 1994, p. 37.

8 Ibid., p. 64-65. La population yougoslave était constituée de six «peuples» : Serbes (36,3\%), Croates (19,7\%), Slovènes (7,8\%), Musulmans (8,9\%), Macédoniens (5,9\%) et Monténégrins (2,5\%) - les trois derniers n’ont été reconnus comme nations qu'à partir du règne de Tito. Le régime reconnaissait aussi trois minorités, soit les Albanais $(7,7)$, les Hongrois $(1,8 \%)$ et les Tsiganes (0,7\%). Environ 5,7\% de la population était considérée «non déterminée» ou «yougoslave». Ces chiffres proviennent du recensement de 1981. Cf. : Catherine Samary, La déchirure yougoslave, op. c it. p. 10. La Yougoslavie était formée de six républiques, dont chacune comporte des minorités. La plus homogène sur le plan ethnique était la Slovénie, peuplée à 90\% de Slovènes. La Serbie n'était peuplée de Serbes qu'à $65 \%$. La Bosnie-Herzégovine n'était constituée que de minorités, les plus importantes étant les Musulmans (au sens 
cède à la résistance antifasciste en tant que préoccupation majeure pour la sécurité commune des peuples yougoslaves. La mise en commun des ressources humaines et matérielles permet un véritable développement économique et social pendant trois décennies. Enfin, la solidarité sociale qu'impose - jusqu'à un certain point - le nouveau régime contribue fortement à maintenir l'unité en dépit des différences nationales et des griefs historiques nombreux.

\section{La Tchécoslovaquie}

$\underline{\text { Retour à la table des matières }}$

Le «divorce de velours» tchécoslovaque a également été perçu sous un angle principalement identitaire, comme si les peuples concernés avaient été unifiés contre leur gré ${ }^{9}$. À l'instar de la Yougoslavie, la Tchécoslovaquie a été créée en 1918 à la suite du démantèlement de l'empire austro-hongrois. En 1914-1915, plusieurs groupes en Bohème-Moravie et en Slovaquie réclamaient l'unification des deux territoires dans un seul État. Il s'agissait là d'une idée alors très populaire. Pour les Tchèques, l'union avec les Slovaques renforcerait leur sécurité face aux Allemands. Parallèlement, l'union avec les Tchèques rassurerait les Slovaques face aux Hongrois. Toutefois, les quelque 20 ans d'existence de cette union n'ont pas permis de tenir les promesses initiales sur le respect de l'égalité des deux peuples. Les Tchèques dominaient les Slovaques comme les Serbes dominaient les autres peuples de la Yougoslavie au cours de la même période. Les deux composantes de la Tchécoslovaquie différaient à maints égards. La Bohème-Moravie se distinguait par un haut degré d'industrialisation et d'urbanisation. La Slovaquie était au contraire principalement agraire, avec les deux tiers de sa population vivant en milieu rural. Ces différences se sont maintenues au cours de l'entredeux-guerres.

ethnique du terme, et non religieux), avec 44\%, les Serbes (31\%) et les Croates (17\%). Ibid., p. 11.

9 «Au plan historique, les Tchèques et les Slovaques ont été unifiés de façon artificielle sur les ruines de l'Empire austro-hongrois ». Extrait d'un éditorial de Michel Audet, Le Soleil, 9 juin 1992, p. A-12. 
En 1939, suite aux pressions des Nazis qui y installèrent un régime fantoche, la Slovaquie devenait indépendante. Néanmoins, tout comme dans la Yougoslavie voisine, la résistance à l'occupation nazie en Bohème-Moravie et en Slovaquie a permis de jeter les bases d'une nouvelle union entre les deux peuples. Cette nouvelle union repose toutefois sur un régime prosoviétique très centralisé dont seul le mouvement populaire de 1968 - le Printemps de Prague - réussira à arracher quelque concession au profit des Slovaques : la transformation de la Tchécoslovaquie en État fédéral. Dans les faits, le caractère fédéral du pays n'était qu'apparent puisque le parti communiste conservait son monopole exclusif dans toutes les sphères d'activité. Cependant, les autorités tchécoslovaques avaient également décidé de mettre fin au retard économique de la Slovaquie.

\section{L'URSS}

$\underline{\text { Retour à la table des matières }}$

L’URSS était l'héritière de la plus grande partie de l'empire tsariste, surnommé «la prison des peuples». Cet empire a été le produit de l'expansion de la Moscovie, dans toutes les directions, mais principalement à l'Est et au Sud. Marc Ferro soutient à juste titre que « la colonisation russe a bien commencé avec la naissance de la nation 10 ». L'Asie centrale fut conquise aux XVIIIe et XIXe siècles aux dépens de la Turquie et de l'Iran. Les républiques caucasiennes provenaient de conquêtes, au XIXe siècle, de territoires sous suzeraineté turque. La Biélorussie et l’Ukraine faisaient partie du premier État russe : la Russie kiévienne, fondée en 862. Elles passeront plus tard sous la domination des Lituaniens et des Polonais. Cette situation amènera la culture et la langue russe des trois futures nations à se distinguer, mais tout en demeurant très apparentées et ce, en dépit du retour à la domination russe qui s'est manifesté par une politique de russification imposée par le tsar, au XIXe siècle.

10 Marc Ferro, « La formation de l'empire russe et soviétique », in Marc Ferro et Marie-Hélène Mandrillon (sous la direction de), Russie, peuples et civilisations, Paris, La Découverte/Poche, 2005, p. 108. 
Après le renversement du régime tsariste, le gouvernement de Lénine, dans la foulée de la révolution d'Octobre, reconnaissait officiellement le droit des peuples à l'autodétermination, ce qui l'avait amené à accepter l'indépendance de la Pologne, de la Finlande et des trois pays baltes. En même temps, la priorité donnée aux objectifs révolutionnaires du régime a mené à la répression des mouvements nationalistes en Géorgie et en Ukraine. Sous Staline, quatre pays ou territoires ont été conquis dans le contexte de la Deuxième Guerre mondiale ou des traités qui l'ont précédée : les trois républiques baltes et la Bessarabie, dont la fusion à la Bucovine a donné naissance à la Moldavie. Les frontières occidentales de l'URSS ont également été modifiées à son profit. Mais ces changements ne concernaient qu'une infime partie du territoire soviétique, qui comptait près de 300 ethnies, dont une centaine reconnues comme minorités par l’État soviétique.

Le monopole politique du Parti et la bureaucratisation du régime faisaient obstacle à toute aspiration nationale. Néanmoins, il faudrait mentionner la réalisation de certains idéaux de la Révolution d'Octobre qui n’ont pas été complètement anéantis par le régime de Staline et de ses successeurs. Même si l'URSS faisait figure d'État hypercentralisé, elle n'en demeurait pas moins, sur le plan de la forme, une fédération de 15 républiques représentant chacune un groupe national important. Les structures politiques et administratives formelles mises en place dans le cadre de cette fédération tendaient à préserver sinon à développer une conscience nationale. La seule existence d'une république d’Ukraine ou d'un Parti communiste de Moldavie entretenait les identités nationales chez les fonctionnaires, intellectuels et professionnels travaillant dans ces structures et aspirant ainsi à une autonomie plus grande, d'où un certain «nationalisme». Le régime soviétique a même procédé au développement de nations qui n’ont jamais eu d'existence propre auparavant, et dont la langue était dénuée de tout alphabet et de toute littérature. Il n'existait pas, avant la révolution d'Octobre, d'État kazakh, ouzbek, tadjik, turkmène ou kirghiz. L'existence de divisions administratives formelles entretiendra le caractère multinational du pays et contribuera à l'éclatement, lorsque le pouvoir central sera affaibli. Comme le rappelle Carol Skalnik Leff : 
Durant la période communiste, dans chaque État ethno-fédéral, le fait que le centre accordait une reconnaissance officielle à l'identité des nationalités titulaires dominantes dans chacune des républiques - identité renforcée par la langue et les droits culturels - a eu des conséquences inattendues pour la mobilisation ethnique. Les spécialistes des nationalités soviétiques ont fréquemment constaté le paradoxe de la politique des nationalités qui, bien que conçue pour mener à l'éventuelle «fusion» des nations, a eu l'effet contraire de fournir des outils pour l'implantation et la consolidation des identités nationales. De plus, les structures fédérales ont fourni des lieux de marchandage politique séparés et distincts pour le développement d'intérêts institutionnels naissants au niveau de la république - ce que Gregory Gleason appelle un «nationalisme bureaucratisé» - et, finalement, une base pour l'émergence d'une élite distinctive au niveau républicain, enracinée dans la nationalité titulaire de cette république. ${ }^{11}$

Pour l'essentiel, l'URSS demeurait une union résultant d'une série de conquêtes plus ou moins anciennes. Seule l'union de la Russie, de l'Ukraine et de la Biélorussie jouissait d'une certaine légitimité sur le plan historique, à défaut d'être confirmée par un régime démocratique.

\section{Les facteurs communs}

$\underline{\text { Retour à la table des matières }}$

L’existence de rancœurs historiques compte parmi les éléments les plus répandus de ceux qui façonnent l'identité et dans les discours qui structurent l'expression du nationalisme. Au Québec, la Conquête de 1760, la répression de l'insurrection de 1837-38, le Rapport Durham, l'exécution de Louis Riel, les référendums sur la conscription, l'imposition de la Loi des mesures de guerre, le rapatriement unilatéral de la Constitution, l'échec de l'entente du lac Meech, constituent autant d'événements qui renforcent l'identité en la distinguant de celle de la majorité anglophone. Les peuples des trois fédérations de l'Est se sont côtoyés plus longtemps et leur histoire est parsemée

11 Carol Skalnik Leff, «Democratization and Disintegration in Multinational States. The Breakup of the Communist Federations», World Politics, 51, Janvier 1999, p. 210. 
d'événements souvent beaucoup plus tragiques. On peut rappeler, dans l'histoire récente, le régime fasciste des Oustachis en Croatie - et auquel s'étaient alliés les Slaves islamisés de Bosnie-Herzégovine -, qui s'est livré au massacre de populations serbes. La répression des multiples nationalités rassemblées dans l'empire russe figure parmi les pages les plus noires de l'époque tsariste. Le régime stalinien a procédé à la déportation de peuples entiers, tels les Tchétchènes, les Ingouches, les Tatars de Crimée. En Ukraine, les nationalistes rappellent la famine de 1933, perçue comme un génocide. Beaucoup moins dramatique, la domination plutôt paternaliste des Slovaques par les Tchèques a été bien réelle pendant plusieurs décennies.

L'oppression nationale peut être invoquée par un certain nombre de peuples des fédérations concernées. Elle l’a été au Québec dans les années 1960 et 1970. La situation socio-économique des francophones se distinguait de celle des anglophones par des revenus inférieurs et un taux de chômage plus élevé, y compris au Québec. La langue anglaise s’imposait souvent comme langue de travail dans les agglomérations urbaines, en particulier à Montréal. L’industrie, le commerce et la finance se trouvaient généralement sous le contrôle des anglophones, qu'ils fussent canadiens, britanniques ou états-uniens.

L'infériorité économique des Slovaques ou des peuples d'Asie centrale par rapport aux Tchèques et aux Russes a été suffisamment documentée. La Slovaquie se distinguait de la Bohème-Moravie par une nette prépondérance de l'agriculture par rapport à l'industrie et, en conséquence, par le poids social et politique de la paysannerie. De cette situation résultait également chez les Slovaques un niveau culturel (taux d'alphabétisation, degré d'instruction, production artistique et littéraire, etc.) inférieur à celui des Tchèques. En URSS, en dépit de l'industrialisation du pays et des progrès remarquables en matière de culture, de santé, de techniques agricoles et autres, les peuples de culture européenne et chrétienne surpassaient ceux d'Asie et de confession musulmane quant aux indicateurs du niveau de vie et de développement. Les inégalités régionales et nationales ont toujours été considérées comme des facteurs propres à alimenter les forces centrifuges. Mais ici s’arrête la comparaison avec le Québec parce que dans le cas des trois fédérations de l'Est, ces facteurs n'ont pas agi de la manière schématique à laquelle certains s'attendaient, c'est-à-dire en provoquant un mouvement en faveur de l'indépendance chez les peu- 
ples qui subissaient la domination économique et qui en payaient le prix le plus élevé sur le plan social. Comme nous le verrons plus loin, la sécession a été l'œuvre des républiques les plus riches, où le niveau de vie était le plus élevé.

\section{L’impact de la transition économique et de la démocratisation}

$\underline{\text { Retour à la table des matières }}$

Les fédérations de l'Est ont connu une transformation des rapports sociaux qui a bouleversé considérablement les relations entre les peuples qui les composaient. Rien de semblable n’a affecté les rapports entre le Canada et le Québec. C’est précisément la politique de décentralisation et d'introduction des rapports marchands pour remplacer partiellement ou totalement l'économie planifiée, suivie de la transition au capitalisme, qui impliquait en particulier la privatisation des entreprises, qui démarquent le plus radicalement le processus qui a mené à l'éclatement des fédérations de l'Est de celui qui alimente le mouvement indépendantiste québécois.

En Yougoslavie, l'introduction des rapports marchands remonte aux années 1960. D’autres pas ont été franchis vers une plus grande libéralisation dans les années 1980, sous l'impulsion de la Ligue des communistes yougoslaves (LCY). C'est donc dire que les changements économiques ont précédé les changements politiques qui menèrent à la fin de la dictature et à l'éclatement du pays, à la fin de 1992. En Tchécoslovaquie, la transition au marché et au capitalisme (les deux volets ont été réalisés de façon simultanée), ne commence qu'après le renversement de la dictature du Parti communiste, en 1989, à l'initiative des forces libérales. L'éclatement du pays ne survient qu'après la démocratisation du régime, soit deux ans plus tard, à la suite de désaccords profonds au sujet de la transition économique déjà amorcée. Enfin, en URSS, la transition politique et le développement des rapports marchands ont été entrepris simultanément, dans le cadre de la perestroïka de Gorbatchev, mais ont précédé l'implosion du pays, à la fin de 1991. Dans tous les cas, la transition économique a donc précédé la disparition des fédérations de l'Est. Que le 
changement ait été amorcé par les partis communistes ou par les forces sociales plus ou moins organisées qui les ont renversés, il n'en demeure pas moins qu'il résulte d'une crise profonde du régime en vigueur dans cette région du monde. Cette crise constitue un élément fondamental du processus qui a mené à l'implosion des États multinationaux de la région.

En Yougoslavie ${ }^{12}$, on l'a vu, l'un des fondements les plus importants de l'Union résidait dans la mise en commun des ressources et leur redistribution au profit des républiques les moins nanties. Pendant quelques décennies, le pays s’est développé rapidement. Néanmoins, du fait de son isolement provoqué par son exclusion du camp soviétique, la Yougoslavie se voyait contrainte à exporter sur les marchés des pays capitalistes, ce qui supposait des entreprises concurrentielles. À partir des années 1960, le modèle yougoslave, fondé sur une forme particulière d'autogestion qui excluait la démocratie à l'échelle de la société, évoluait donc graduellement vers l'économie de marché et la décentralisation. Le revenu national commençait ainsi à dépendre des ventes sur les marchés internationaux. Cette dynamique a incité les républiques les plus riches à s'opposer de plus en plus à un régime fédéral qui les contraignait au transfert de ressources vers les républiques plus pauvres. À ce titre, la Slovénie, république très industrialisée, et la Croatie, dont l'économie était déjà fortement axée sur le tourisme et les devises fortes qui en résultaient, bénéficiaient d'une position favorable sur le marché mondial. Par ailleurs, elles se plaignaient également de la surreprésentation des Serbes dans les institutions de l'État. Les républiques riches se sentaient donc "exploitées » par un régime qui imposait une certaine répartition de la richesse vers les régions plus pauvres. Le régime lâchera du lest en 1974 en accordant une autonomie encore plus large aux républiques - la Yougoslavie se définissant dès lors comme une confédération -, mais tout en préservant le monopole politique de la LCY. Sur le plan économique, le caractère confédéral du régime était bien réel puisque les républiques pouvaient conserver une plus grande part des ressources, au détriment de l’État fédéral et, donc, des républiques plus pauvres.

12 Notre analyse de l'ex-Yougoslavie emprunte largement aux travaux de Catherine Samary, résumés dans son ouvrage intitulé : La déchirure yougoslave, op.cit. 
La confédération a eu pour effet de réduire considérablement la solidarité économique et ainsi d'éloigner les différentes communautés nationales les unes des autres. Dans les années 1980, les politiques économiques libérales appliquées en réaction à la crise économique allaient aggraver le problème. La décentralisation donnait ainsi un élan aux forces nationalistes qui ont rapidement conclu que l'indépendance leur apporterait davantage, ayant désormais l'opportunité de mettre la main sur les richesses à privatiser. En 1991, la Slovénie déclarait son indépendance, ensuite imitée par la Croatie. Se sentant menacée par la prépondérance de la Serbie nationaliste de Slobodan Milosevic dans ce qui restait de la Yougoslavie, la BosnieHerzégovine fera sécession l'année suivante.

Comme l'a rappelé Catherine Samary, les périodes de grande cohésion dans l'histoire de la Yougoslavie se caractérisaient par une amélioration du niveau de vie et des droits des nationalités ${ }^{13}$. À l'inverse, la rupture a été préparée par la crise socio-économique et politique des années 1980, et non par les prétendues haines interethniques, qui ont toutefois fini par ressurgir :

À partir de 1980, la crise économique a remis en cause la croissance permanente du niveau de vie, observée depuis 1945. Or, ce mieux-être contribuait à rendre légitime l’État commun. Parallèlement, le compartimentage économique croissant des républiques a aggravé les écarts de développement, soulignant le fait que chaque bureaucratie du parti-État fondait de plus en plus ses sources de pouvoir et de privilèges sur des bases nationales, républicaines ou provinciales ${ }^{14}$.

À cela s'ajoute une dimension proprement politique : à l'instar de ce qui se passera par la suite en URSS, les premières élections libres, tenues en 1990, ont été organisées à l'échelle de chacune des républiques nationales. La république devenait ainsi l'espace démocratique par excellence et en conséquence jouissait d'une légitimité qui surclassait celle des autorités " confédérales » de la Yougoslavie, issues de la dictature de la LCY.

13 Catherine Samary, La déchirure yougoslave, op. cit., p. 24.

14 Jean-Arnault Dérens et Catherine Samary, Les conflits yougoslaves de A à Z, op. cit., p. 10-11. 
Le développement accéléré des rapports marchands a donc eu pour effet d'alimenter des forces centrifuges dans les républiques les plus riches, la Slovénie et la Croatie, d'où est venue l'initiative indépendantiste. Il s'agit là d'une différence notable avec la situation qui caractérise le conflit Canada/Québec. Ce que Catherine Samary désigne comme la mentalité du « chacun pour soi »15, ou ce que Hobsbawm appelle "l'égoïsme collectif de la richesse » 16 a ainsi prévalu. Ce phénomène n'épouse pas forcément les seuls contours d'une nation ou d'un groupe ethnique. Certains mouvements politiques du nord de l'Italie et de la Flandre belge affichent la même attitude séparatiste à l'égard des régions plus pauvres. La chose a été maintes fois observée dans les banlieues plus cossues des grandes villes - comme Montréal - qui refusent de fusionner avec la ville principale pour éviter tout transfert de richesses à leurs dépens.

Il s'avère donc impossible de comprendre les conflits nationaux du seul point de vue des nationalismes en concurrence les uns avec les autres. D'ailleurs, le peuple slovène était celui qui, à tous égards, avait le moins manifesté de sentiments nationalistes au cours des six décennies d'existence de la Yougoslavie. La Slovénie n’a jamais eu d'existence comme État avant le régime de Tito. Même sous la domination austro-hongroise, les Slovènes n'ont pas revendiqué d'autonomie territoriale ${ }^{17}$. Néanmoins, ils furent les premiers à déclarer leur indépendance. En juin 1991, la tentative de l'armée serbe pour empêcher la sécession de la république aura évidemment pour effet de renforcer considérablement le sentiment nationaliste en Slovénie. Mais il n'en demeure pas moins que ce n'est pas l'idéologie nationaliste qui a provoqué le changement : il faut d'abord expliquer pourquoi l'idéologie nationaliste a fini par prendre une place importante dans la vie politique slovène.

C'est ainsi que l'on peut comprendre que la transition à l'économie de marché et, plus tard, au capitalisme, a donné le coup de grâce à la Yougoslavie en alimentant le désir de la Slovénie et de la Croatie d'arrimer leurs économies à celle de l'Union européenne pour en tirer

\footnotetext{
15 Ibid., p. 65.

16 Eric J. Hobsbawm, L'Âge des extrêmes. Histoire du Court XXe Siècle, Bruxelles, Éditions Complexe, 2003, p. 555.

17 Ibid., p. 38.
} 
tous les bénéfices, débarrassés de l'obligation de partage avec les républiques et provinces plus pauvres. En même temps, on pouvait aussi s'émanciper de la domination exercée par les Serbes dans les institutions de l’État.

La Tchécoslovaquie, comme nous l'avons mentionné plus haut, n’a éclaté qu'après la prise du pouvoir par les libéraux et qu'après qu'a été amorcé le développement des rapports marchands et la privatisation. Les conflits entre les futures républiques tchèque et slovaque ont pris de l'ampleur au cours des années 1990 à 1992, celles où ont eu cours les débats sur le rythme et les modalités de la transition à l'économie de marché capitaliste. Les raisons économiques y surpassent toutes les autres et de manière plus évidente qu'ailleurs.

Au moment où la Tchécoslovaquie entreprend les transformations économiques, politiques et sociales consécutives à la Révolution de velours de novembre 1989, les deux entités qui la composent diffèrent considérablement sur le plan économique et commercial. Les années qui ont suivi la répression du Printemps de Prague ont été en partie consacrées à l'industrialisation de la Slovaquie, qui accusait un retard important par rapport à la Bohème-Moravie. Le régime a donc procédé à des investissements massifs, principalement dans l'industrie lourde et d'armement. Des usines de taille considérable ont été construites. Par comparaison, la Bohème-Moravie comptait un très grand nombre de PME. Le paysage agricole des deux entités différait tout autant, celui de la Slovaquie étant particulièrement marqué par les grandes monocultures. Par ailleurs, la Slovaquie avait pour principal marché d'exportation l'Union soviétique et les autres pays membres du Pacte de Varsovie.

Ces différences laissaient présager que les conséquences économiques et sociales liées à l'application des mêmes recettes pro-marché seraient plus dramatiques en Slovaquie qu'en Bohème-Moravie. D’ailleurs, l'effondrement de l'URSS et le passage accéléré au capitalisme en Pologne et chez les autres pays voisins ont eu pour effet de réduire considérablement les exportations slovaques. Le taux de chômage est rapidement passé de 0 à $12 \%$ alors que la Bohème-Moravie, 
beaucoup moins affectée, a connu une augmentation plus légère, de 0 à $2,9 \% 18$.

L'une des priorités consistait à convertir l'industrie d'armement, mais cela prendrait plusieurs années. La privatisation de l'économie slovaque s'annonçait forcément plus lente ou plus complexe qu'en Bohème-Moravie, compte tenu de la taille des entreprises. La classe politique slovaque en a promptement conclu que la restructuration économique - en rappelant l'exemple de l'Allemagne d'Adenauer devait s'effectuer sous la direction de l'État et non de la main invisible du marché, comme le soutenait l'équipe d'économistes de l'École de Chicago réunie autour du premier ministre tchèque Vaclav Klaus.

Le «divorce de velours » ne résulte donc pas simplement de sentiments nationaux, de rancœurs historiques ou d'ambitions nationales insatisfaites : il est le produit direct d'un désaccord majeur au sujet du rôle de l'État dans la transition, du rythme des transformations et de leurs conséquences sur la population. Après avoir en vain réclamé une plus grande autonomie au sein de la République fédérale tchèque et slovaque 19 - une sorte de "souveraineté-association » 20 - le gouvernement slovaque se voyait imposer deux possibilités par le gouvernement tchèque : une fédération centralisée ou l'indépendance. Les libéraux tchèques entrevoyaient d'ailleurs d'un bon œil une séparation de la Slovaquie, puisqu'elle leur permettrait, pensaient-ils, d'intégrer plus rapidement la république tchèque à l'Union européenne, de ne plus avoir de frontières communes avec l'ancienne URSS et d'éviter de dépenser des ressources pour la conversion de l'industrie d'armement, principalement concentrée en Slovaquie. Cette attitude des dirigeants tchèques a été qualifiée par certains de " nationalisme débarrassiste » ${ }^{21}$. Il est important de souligner ici que les enquêtes d'opinion effectuées auprès des populations tant tchèque que slovaque révélaient une opposition majoritaire à la séparation des deux entités. En Slovaquie, moins du tiers se disait favorable à l'indépendance, une

18 Claire Wallace, «Citizenship and Social Policy in East-Central Europe», in Marguerite Mendell and Klaus Nielsen (ed.), Europe: Central and East, Montreal/New York/London: Black Rose Books, 1995, p. 283.

19 C’est le nom que prend officiellement le pays le 20 mai 1990.

20 Le gouvernement slovaque de Vladimir Meciar ne voulait concéder au gouvernement fédéral que la politique étrangère et la fiscalité.

21 Catherine Samary, La déchirure yougoslave, op. cit., p. 129. 
proportion plus faible encore chez les Tchèques. Toutefois, il n'existait pas d'organisations politiques suffisamment fortes pour empêcher la sécession et jeter les bases d'un nouvel État qui aurait accommodé les deux parties 22 .

Les dirigeants tchèques et slovaques se sont d'ailleurs abstenus de procéder à une consultation populaire sur le sujet, même si la majorité des Tchèques et des Slovaques considérait le référendum comme un moyen plus légitime pour décider de l'avenir de leur union. En revanche, il est également vrai que la séparation n’a pas suscité de mouvement de masse contre elle. "Ce fut donc une impuissance civique, caractéristique de la culture politique antérieure, qui mena à l’indépendance des deux États. Et la décision du Parlement fédéral fut acceptée sans résistance 23 ». Les assemblées législatives des deux futurs pays indépendants ont approuvé la séparation, devenue effective le 31 décembre 1992.

La fédération tchécoslovaque a éclaté trois ans après les changements politiques qui ont mené à la démocratisation du pays. En ce sens, la dictature ne peut expliquer à elle seule le maintien de l'intégrité du pays. L'absence même de mobilisation populaire - de part et d'autre - pour mettre fin à l'union exclut également d'éventuelles rancœurs historiques comme causes possibles du «divorce de velours». Ce sont bel et bien des divergences fondamentales sur le rythme et les modalités de la transition qui ont mené à la sécession, qui résulte d'une entente entre les deux gouvernements concernés.

Les causes de l'éclatement de l'URSS sont multiples et varient d'une république à l'autre. Il ne s'agit pas tant - à quelques exceptions près - de nationalismes exprimés à l'égard d'un peuple désigné comme oppresseur ou privilégié mais d'une lutte menée contre le gouvernement de l'Union. Toutes les républiques et toutes les régions de l'URSS s'estimaient exploitées par le Centre, y compris la République socialiste fédérative soviétique de Russie (RSFSR). Des motifs économiques, politiques et environnementaux ont alimenté les forces

22 Martin Bùtora et Zora Bùtorova, «Identités en transition : de la Tchécoslovaquie à la Slovaquie», Politique et Sociétés, no. 28, $14^{\mathrm{e}}$ année, automne 1995, p. 120.

23 Ibid., p. 121. 
centrifuges bien davantage que la seule altérité. Autrement, comment la séparation des peuples slaves, pour ne pas mentionner l'appui des minorités russes à l'indépendance des républiques non-russes deviendrait-elle intelligible ? Comme l'a écrit Jacques Sapir, l'Union soviétique a éclaté non seulement parce qu'elle était un empire, mais aussi parce qu'elle était un système ${ }^{24}$. Si les arguments identitaires suffisaient en eux-mêmes, il faudrait alors expliquer pourquoi les républiques d'Asie centrale, peuplées de musulmans parlant des langues apparentées au turc et au persan, ont été les dernières à accepter cette option.

Comme en Yougoslavie, la volonté d'indépendance s'est d'abord manifestée dans les républiques bénéficiant du niveau de vie le plus élevé de l'URSS. Les républiques les plus pauvres - situées en Asie centrale - ont été placées devant le fait accompli de la fin de l'union. Mus par un «égoïsme collectif de la richesse» et un "nationalisme débarrassiste » du même type que celui qui a été à l'œuvre en Slovénie, en Croatie et en République tchèque, les libéraux russes n’ont pas voulu de ces territoires perçus comme un fardeau pour la Russie.

L'introduction des rapports marchands en URSS jouera un rôle de première importance dans l'éclatement du pays. Le système économique soviétique, caractérisé par la propriété étatique des moyens de production, la planification centralisée et le monopole d’État du commerce extérieur, conséquence du pouvoir absolu exercé par l'appareil du Parti communiste, était devenu inapte à assurer l'amélioration du niveau de vie de la population et le maintien du statut de superpuissance de l'URSS. Des réformes apparaissaient de plus en plus nécessaires au lendemain de la mort de Léonide Brejnev, en novembre 1982. La nomination de Mikhaïl Gorbatchev au poste de secrétaire général du PCUS en mars 1985 allait inaugurer un processus de réformes destiné à remédier aux défauts du système soviétique. Le nouveau secrétaire général souhaitait faire sortir l’Union soviétique de la « stagnation » dans laquelle elle était plongée depuis le milieu des années 1970 en redynamisant la société. La réforme économique se situait au cœur du programme, mais la nouvelle équipe réformiste avait compris que son succès dépendait d'autres mesures, d'ordre politique, pour secouer les couches les plus conservatrices de la bureaucratie et

24 Jacques Sapir, Feu le système soviétique?, Paris, La Découverte, 1992, p. 12. 
mobiliser la population derrière le gouvernement. L'expérience des réformes précédentes avait enseigné qu'à défaut de recourir aux méthodes staliniennes pour imposer la volonté des autorités centrales, tout projet de changement qui laisserait intact le caractère bureaucratique du régime serait voué à l'échec, compte tenu de la méfiance des ouvriers et employés qui faisaient généralement les frais de ces réformes, et de l'hostilité de certaines franges de l'administration ou des directions d'entreprises qui craignaient les changements de routine susceptibles de menacer leurs privilèges.

Pour ce faire, il comptait effectuer une refonte du système économique - ou perestroïka - et mettre de l'avant une politique de transparence (glasnost) destinée à démontrer à l'ensemble de la société soviétique que, désormais, il fallait tenir le «langage de la vérité». Les médias ont ainsi été invités à critiquer différents aspects du régime et les historiens pouvaient entreprendre la révision de l’histoire.

Pour l'essentiel, le but de la réforme économique consistait à mettre fin aux pénuries et à encourager l'innovation technologique en accordant une autonomie accrue aux dirigeants des entreprises et en impliquant les travailleurs dans la gestion. En incitant les entreprises à faire des bénéfices et à en utiliser une partie en fonction de leurs priorités (nouveaux investissements, amélioration des conditions de travail, etc.), les réformateurs gorbatchéviens s'attendaient à libérer l'initiative dans toute la chaîne de commandement. En permettant aux entreprises de choisir leurs fournisseurs, on espérait une amélioration marquée de la qualité des biens destinés aux consommateurs. Mais les résultats attendus n'ont pas été au rendez-vous. L'autonomie accrue des entreprises n'a eu aucun effet positif sur la production, tant sur le plan quantitatif que qualitatif. La liberté nouvelle accordée à des monopoles a au contraire incité les entreprises à diminuer la production sans qu'elles en soient pénalisées puisqu'elles compensaient les pertes par des hausses de prix ou par un transfert des biens produits vers les réseaux de distribution privés en émergence. Du côté des travailleurs, les nouvelles mesures les rendaient en partie responsables des difficultés, ce qui a eu un effet direct sur leurs revenus, l'État ayant décidé d'éliminer l'accès à certains services, jusque-là gratuits. Par ailleurs, les travailleurs figuraient parmi les premières victimes de l'aggravation des pénuries. Bref, tout cela a eu un effet désastreux sur 
la productivité du travail et a fini par braquer les travailleurs contre Gorbatchev et l'État central.

Dans la mesure où l'État fédéral soviétique concentrait tous les pouvoirs entre ses mains, il devenait ainsi le point focal vers lequel convergeaient toutes les récriminations. Le système économique était à la fois hypercentralisé et déconcentré : l'immensité du pays et la complexité de l'économie impliquaient des directives très générales imposées par les autorités centrales mais, en même temps, l'application de ces directives et leur désagrégation en directives précises et opérationnelles laissaient beaucoup d'autonomie aux autorités républicaines et locales. Le caractère fédéral de l'URSS allait donc au-delà de la simple apparence. L'échec de la perestroïka aura pour effet d'aggraver les pénuries et donc de réduire la quantité de ressources distribuées par le Centre. Dans un tel contexte, le secrétaire du parti des entités régionales (qui représente le pouvoir central) aura moins d'autorité et les frustrations des dirigeants républicains prendront parfois un caractère nationaliste 25 .

Par ailleurs, l'autonomie accrue des entreprises, à laquelle s’ajoutaient d'autres mesures comme la légalisation du marché noir et le droit de créer des petites entreprises privées et des coopératives, ont favorisé la croissance de nouvelles forces sociales qui aspiraient à se détacher complètement de la tutelle de l'État et, éventuellement, à restaurer la propriété privée et les rapports marchands. Le gouvernement central soviétique constituant le principal obstacle à ces aspirations, les représentants de ces nouvelles forces sociales s'éloignèrent de Gorbatchev et se replièrent sur les instances républicaines, pour des raisons sur lesquelles nous reviendrons plus loin.

La glasnost permettait à tous les mécontentements de s'exprimer. Alors qu'elle était destinée au départ à mobiliser la population contre les forces opposées aux réformes, elle se tourne rapidement contre ceux qui l'ont instaurée en donnant des moyens d'expression à ceux qui jugent le changement trop timoré. Qui plus est, les manifestants et opposants craignaient de moins en moins les représailles, Gorbatchev étant devenu l'apôtre du désarmement et de la résolution des conflits régionaux dans le monde. Le recours à la répression massive aurait

25 Ibid., p. 18. 
terni la réputation internationale de Gorbatchev et renforcé les conservateurs du Parti, qu'il combattait.

La glasnost impliquait également la révision de l'histoire. Ici encore, l'intention première des dirigeants réformistes consistait à asseoir la légitimité de leurs réformes dans la poursuite de la déstalinisation interrompue après la mise à l'écart de Khrouchtchev. Il s'agissait, entre autres, de justifier le virage pro-marché par une réhabilitation des compagnons de Lénine qui ont soutenu la Nouvelle politique économique 26 (dont Gorbatchev disait s'inspirer) jusqu'au bout, avec, en tête, l'économiste bolchévique Nicolaï Boukharine. La révision de l'histoire aura vite fait de sortir du cadre de l'autojustification des politiques de Gorbatchev. Les républiques baltes, qui conservaient la mémoire encore récente de leur indépendance et de la manière dont Staline les avait intégrées à l'Union soviétique, ont pu prouver, avec toute la documentation nécessaire, leur annexion par la force en 1940, conformément à une clause secrète du Pacte Ribbentrop-Molotov, réfutant la version officielle du régime soviétique au sujet de leur adhésion prétendument volontaire à l'Union. Du reste, la perestroïka impliquait également l'expérience d'une politique d'autonomie économique régionale dans ces trois républiques, alimentant ainsi le mouvement d'appui à la réforme tout en en révélant les limites ${ }^{27}$. Par leur situation géographique, la Lituanie, la Lettonie et l'Estonie ont conclu qu'elles tireraient avantage à amarrer leurs économies à celles des pays scandinaves et de l'Union européenne. En très peu de temps, les forces autonomistes, encouragées par les changements politiques, ont ouvertement opté pour l'indépendance. Leur inclusion dans l’Union étant illégitime, elles réclamaient le droit de la quitter et de reprendre leur statut d'États indépendants.

26 La Nouvelle politique économique ou NEP a été en vigueur de 1921 à 1928. Elle consistait, dans l'essentiel, à accorder une certaine liberté aux petites et moyennes entreprises privées et à instaurer des rapports d'échange entre la campagne et la ville, par opposition aux réquisitions forcées effectuées au cours de la guerre civile (1918-1921).

27 On pourra consulter à ce sujet, Sarah Ginaite, «La réforme économique et les perspectives d’indépendance économique des républiques baltes», et Algis Sileika, «La conception de l’autonomie économique d’une république fédérée de l'URSS et les problèmes de sa mise en pratique», in David Mandel (sous la direction de), La perestroïka: économie et société, Sillery, Presses de l’Université du Québec, 1990, p. 199-208 et 209-217. 
L'affaiblissement de l'aile conservatrice du Parti et l'émergence de mouvements populaires en faveur de l'accélération des changements politiques amènent plusieurs alliés de Gorbatchev à rompre avec lui, ayant décidé qu’il fallait désormais démocratiser entièrement le régime et passer intégralement à l' «économie de marché ». Avec l'approfondissement de la crise économique, sociale et politique, ces libéraux autoproclamés "démocrates » trouvèrent de larges échos auprès des masses. Par ailleurs, ces promoteurs de «réformes radicales» appuyaient généralement les mouvements nationalistes à l'œuvre dans les républiques. D'où une sorte d'alliance entre ceux qui prônaient la démocratisation du régime, le passage à l'économie de marché (sans toutefois définir de quoi il s'agissait) et le respect des droits des nationalités.

En somme, le conflit entre les forces «réformistes» et «conservatrices» prenait la forme d'une lutte des républiques contre le gouvernement central de l'Union. Cette lutte politique pour une accélération de la libéralisation économique et de la démocratisation bénéficiait également d'un soutien populaire assez répandu dans plusieurs républiques. Le nationalisme des élites rejoignait donc celui des peuples, même si c'était souvent pour répondre à des aspirations différentes. Chez les élites, le nationalisme constituait un moyen d'accroître leur pouvoir et leurs privilèges; c'était aussi un moyen pour canaliser la colère populaire contre le pouvoir central (l'Union) ou contre les minorités; enfin, il s’agissait là du moyen idéal pour demander au peuple de consentir aux sacrifices qu'une transition au capitalisme potentiellement impopulaire exige. Chez les peuples, le nationalisme était mû par des considérations démocratiques dont l'aspiration à un accroissement du contrôle local sur l'économie, la volonté de protéger l'environnement - ce dont le pouvoir central s'était révélé incapable en le saccageant sans la moindre opposition. C'est pourquoi les environnementalistes se sont également inscrits dans la mouvance visant à accroître les pouvoirs des autorités des républiques, considérées plus proches des citoyens. On peut ainsi comprendre la montée éphémère du nationalisme en Biélorussie. Le 26 avril 1986 a eu lieu le désastre de Tchernobyl. Plus de $70 \%$ des retombées radio-actives se sont concentrées dans la seule Biélorussie. C'est alors que l'opposition anti-soviétique a pris son envol, comme ce fut également le cas en Ukraine, où se trouve la ville de Tchernobyl. Sans aller aussi loin 
qu'en Biélorussie, les griefs de nature environnementale à l'égard du gouvernement soviétique ont également nourri les rancœurs chez les peuples d'Asie centrale. Les Kazakhs lui reprochent d'avoir systématiquement empoisonné leur environnement dans les 40 années qui ont suivi la Deuxième Guerre mondiale 28.

Dans le contexte réformiste des années 1989-1990, les pressions devenaient de plus en plus fortes pour que Gorbatchev procède à une dévolution du pouvoir aux instances républicaines, perçues comme étant mieux en mesure de répondre aux aspirations des citoyens, qu'elles soient d'ordre économique, environnemental ou culturel. Ce repli sur les républiques contribuera à engendrer une sorte de démocratisation asymétrique. Les réformes de Gorbatchev impliquaient une démocratisation partielle du système politique, dont l'élection des députés dans un cadre compétitif mais tout en interdisant la pluralité des partis au profit du seul Parti communiste. Les députés, à leur tour, devaient désigner le président de l'URSS. Ces élections semi-libres ont eu lieu en 1989. Or, la rupture des réformistes plus radicaux avec Gorbatchev les a incités à se replier sur les instances républicaines. Les élections des députés et présidents des républiques fédérées ont eu lieu l'année suivante, en 1990, alors que le mouvement pour la démocratisation du régime et les débats sur l'avenir de l'URSS avaient progressé de façon marquée. Ces élections se révélaient déjà beaucoup plus compétitives, pluralistes et démocratiques que celles qui avaient permis de procéder à la désignation des membres du Congrès des députés du peuple de l'URSS. Cette démocratisation asymétrique sera amplifiée en juin 1991 par l'élection au suffrage universel direct du président de la RSFSR (la future Russie), Boris Eltsine, dont la légitimité surpassera alors celle de Gorbatchev.

Des aspects plus «identitaires» militaient également en faveur d'un repli sur les républiques, telle la protection de la langue et de la culture. Dans certains cas, il s'agissait également d'une réaction au statut privilégié de la langue russe à l'échelle de l'Union. À titre d'exemple, la primauté du tadjik sur le russe n’a été garantie par une loi qu'en 1989. Au Turkménistan, le turkmène ne devenait l'égal du russe qu'en

28 Martha Brill Olcott, «Kazakhstan : pushing for Eurasia», in Ian Bremmer and Ray Taras (ed.), New States, New Politics: Building the Post-Soviet Nations, Cambridge: Cambridge University Press, 1997, p. 505. 
mai 1990. Le russe constituait également la langue de l'administration au Kazakhstan, jusqu'à ce que les revendications des Kazakhs à l'époque de la perestroïka aboutissent à une amélioration du statut de leur langue.

En Géorgie, l'identité a certes joué un rôle plus important que l'économie. Le pays a connu une brève période d'indépendance entre 1918 et 1921. Par la suite, il a été brutalement reconquis par la Russie soviétique. Le nationalisme géorgien s'est exprimé à différentes reprises au cours de la période soviétique, notamment en réaction à la politique de russification. Par exemple, la Constitution de 1977 faisait de la langue russe la langue officielle de la Géorgie, au même titre que le géorgien. La Géorgie se caractérise entre autres par la coexistence de fortes minorités, dont les Abkhazes, les Ossètes du Sud et les Adjars, situation qui alimente l'insécurité démographique de la majorité géorgienne. Le combat contre le statut privilégié de la langue russe a donc pris la forme d'une politique destinée à renforcer la culture et la langue géorgienne, souvent au détriment de celles des minorités. En se tournant contre les minorités, le nationalisme géorgien a pavé la voie à un régime autoritaire dès 1990.

En Moldavie, il s'agissait clairement d'un mouvement nationaliste alimenté par des facteurs liés à l'identité. Une partie du territoire, la Bessarabie, avait été conquise dans le cadre du Pacte RibbentropMolotov. La mémoire de la collectivisation forcée de ce territoire, à la fin des années 1940, était encore vive. Les Moldaves avaient également subi une campagne de russification qui les avait forcés à utiliser l'alphabet cyrillique, alors que leur langue est proche du roumain. Les minorités russe et ukrainienne dominaient l'économie urbaine. Le niveau de vie de la Moldavie était le plus faible de toutes les républiques non asiatiques de l'URSS. Donc, un statut économique inférieur, une minorité qui domine dans les centres urbains, la langue russe dominante dans les milieux politiques et scientifiques, tout cela peut rappeler les facteurs qui ont présidé à la montée du nationalisme québécois dans les années 1960 .

Mais ces aspects liés à l'identité ne sauraient expliquer à eux seuls les multiples griefs adressés au pouvoir central et la volonté de s’en éloigner, sans forcément rompre tout lien avec le gouvernement soviétique et les républiques fédérées. Comme l'expliquent Alexander Motyl et Bohdan Krawchenko, si on fait abstraction de certaines régions 
de l'ouest de l'Ukraine, les facteurs socio-économiques l'emportent sur les facteurs identitaires :

Le système bureaucratique centralisé était vu comme un frein au développement économique et social et à la modernisation. Moscou n'avait rien à offrir - ce n'était ni une source de savoir-faire technologique, ni un centre financier international. C'était simplement un appareil de répression et de contrôle ${ }^{29}$.

Gorbatchev devait donc impérativement, sous la pression populaire et celle des élites des différentes républiques, redéfinir les rapports entre le pouvoir central et les membres de la fédération. Un référendum eut lieu le 17 mars 1991 et posait la question suivante :

« Estimez-vous nécessaire de préserver l’Union des républiques socialistes soviétiques en tant que Fédération rénovée de républiques constitutionnellement souveraines et égales dans laquelle les droits de la personne et les libertés seront pleinement garantis pour les gens de toutes les nationalités?»

Le référendum a été boycotté par les trois républiques baltes, la Géorgie, la Moldavie et l'Arménie, là où l'option indépendantiste était la plus forte. Mais dans les neuf autres républiques - les plus peuplées - le taux de participation a atteint les $80 \%$. Même si les libéraux et les nationalistes appelaient à l'annulation, plus des trois quarts $(76,4 \%)$ des participants ont refusé de les suivre sur cette question et ont appuyé le projet de Fédération rénovée. En Ukraine, le gouvernement nationaliste avait ajouté une question supplémentaire sur la souveraineté de la république. C'est ainsi que les Ukrainiens ont voté à la fois en faveur de l'Union (75\%) et en faveur de la souveraineté (90\%)! Contrairement à ce qui a fini par s'imposer dans le vocabulaire politique québécois, les concepts de «souveraineté» et d' " indépendance » n'avaient pas le même sens dans une URSS sur le point de succomber. La souveraineté signifiait bel et bien que c'est par rapport au pouvoir central qu'on désirait prendre ses distances, et non par rapport aux autres républiques de l'Union. On acceptait que certains pouvoirs

29 Alexander Motyl et Bohdan Krawchenko, «Ukraine : from empire to statehood», in Ian Bremmer and Ray Taras (ed.), op. cit., p. 244. 
soient délégués à l'Union, mais tout en conservant la possibilité de les lui retirer.

Dans les mois qui ont suivi, le déclin économique avait atteint un tel degré que tous les peuples de toutes les républiques voulaient se débarrasser totalement du gouvernement central, considéré comme l'unique responsable de la crise. Le «déficit de légitimité» du centre par rapport aux républiques amenait les autorités de ces dernières à refuser les lois de l'Union non conformes à leurs intérêts, allant même jusqu' à refuser de verser leurs contributions au budget fédéral. Tout cela s'est déroulé dans un contexte d'aggravation des pénuries qui a fait perdre tout attrait au rouble. Désormais, mieux valait contrôler les biens matériels que d'accumuler du papier-monnaie rongé par l'inflation ${ }^{30}$. Ce processus de désagrégation affectait non seulement les républiques, mais également les villes (par exemple, en réservant l'accès aux magasins aux seuls citoyens de la ville) et les entreprises. Encore une fois, le facteur économique, lié à la crise du régime bureaucratique, au développement des rapports marchands et à la transition au capitalisme demeure incontournable.

Tout cela explique que même les minorités russes d'Ukraine et des républiques baltes ont voté en faveur de l'indépendance. Il s’agit là d'une autre différence majeure par rapport au Québec où les minorités anglophones et allophones appuient massivement le maintien du régime fédéral canadien. Au Kazakhstan, les Kazakhs ne formaient que $39.5 \%$ de la population (recensement de 1989) contre 37.7 pour les Russes ${ }^{31}$. Les gouvernements des républiques européennes de l'URSS étaient perçus comme étant plus démocratiques et donc, plus contrôlables. L'indépendance devenait ainsi un moyen plus sûr d'accélérer la transformation économique, politique et sociale. Mais cela ne signifie pas pour autant, hormis pour les six républiques n’ayant pas participé au référendum, que les peuples concernés voulaient mettre fin à toute union.

30 Jacques Sapir, op. cit. p. 18-19.

31 Les peuples slaves et slavisés du Kazakhstan formaient 50 \% de la population totale de la république. Martha Brill Olcott, «Kazakhstan : pushing for Eurasia», in Ian Bremmer and Ray Taras (ed.), op. cit., p. 547. 
Comme l'a remarqué Hobsbawm, «la 'conscience nationale' se développe de façon inégale parmi les groupes sociaux 32 ». Lorsque l'effondrement du régime soviétique apparaîtra comme une éventualité, le nationalisme, chez les élites, deviendra un instrument pour accroître leurs pouvoirs et privilèges, surtout lorsqu'il s'agira de s'emparer des richesses. En effet, le passage de la propriété d'État à la propriété privée signifiait que l'essentiel des richesses de l'Union soviétique cherchait preneurs. Si l'on s'en tient au projet péquiste - et en cela il se distingue de la frange plus à gauche du mouvement indépendantiste passé et actuel (RIN, FLQ, Parti-pris, Québec solidaire, etc.) - la souveraineté du Québec ne modifierait pas fondamentalement le sort des couches les plus privilégiées de la population, dans la mesure où le régime social demeurerait le même. Par contre, en URSS, les richesses principales du pays étaient disponibles. À titre d'exemple, l'indépendance de l'Ukraine a, dans les faits, servi à renforcer le contrôle de la nomenklatura locale sur le pays. Selon certaines estimations, plus de 95\% des actifs industriels d'Ukraine appartenaient aux ministères centraux de l'URSS. Si des liens politiques avaient été maintenus avec la Russie, la propriété aurait été partagée avec Moscou. Tel ne fut pas le cas : la nomenklatura a réussi non seulement à mettre la main sur la propriété et des ressources économiques vastes mais elle a également réussi à faire tout cela libre de tout contrôle, de toute supervision démocratique. C'est ce qui explique l'alliance entre l'organisation nationaliste ukrainienne - le Roukh - et le Parti communiste d'Ukraine, dirigé par l'antinationaliste Léonide Kravtchouk. Cette dimension - le contrôle des richesses - est absente au Québec, à tout le moins sous cette forme. D'ailleurs, comment comprendre autrement que, dans plusieurs républiques de l'ex-URSS, la bourgeoisie en devenir favorisait l'indépendance alors qu'au Québec, les milieux d'affaires se disent très majoritairement fédéralistes, comme le prouve la position des grands lobbys patronaux ? En Russie, en Ukraine et ailleurs, les forces pro-capitalistes étaient indépendantistes 33 .

32 Eric J. Hobsbawm, Nations et nationalisme depuis 1780. Programme, mythe, réalité, Paris, Gallimard, 1992, p. 31.

33 Ironie de l'histoire : la Russie indépendante, qui se considérait - au même titre que les autres - victime d'un régime oppresseur exerce aujourd'hui une domination économique et politique dans tout l'espace postsoviétique. La redistribution des richesses a été remplacée par l'utilisation des ressources 
Il existe certes des républiques où ces dimensions liées à la transition économique ont été occultées par d'autres. En Arménie, le nationalisme s'est manifesté dans le contexte de la remise en cause des frontières héritées du régime soviétique. La liberté nouvelle permise par la glasnost a donné l'occasion aux habitants du NagornyKarabakh, territoire enclavé dans la république soviétique d'Azerbaïdjan mais peuplé à plus de 75\% d'Arméniens, d'exprimer leur volonté de rattachement à l'Arménie voisine. Cette revendication est à l'origine du conflit armé qui a opposé les deux républiques depuis $1988{ }^{34}$. Ce conflit a donné lieu à des pogroms commis par des Azerbaïdjanais (apparentés aux Turcs ${ }^{35}$ ) à l'encontre d'Arméniens résidant en Azerbaïdjan - notamment à Soumgaït en 1988 - et à une vague d'expulsions et de migrations de part et d'autre de la frontière ${ }^{36}$, ravivant ainsi, du côté de l'Arménie, la mémoire du génocide perpétré par la Turquie en 1915. Jusque là, l'appartenance à l'URSS était perçue par les Arméniens comme un moindre mal face à une éventuelle domination turque. Ne se sentant plus protégés par le gouvernement de l'Union - qui semblait prendre le parti des Azerbaïdjanais en refusant de modifier les frontières - les Arméniens ont finalement évolué vers le sécessionnisme. Par ailleurs, les griefs à l'égard du gouvernement soviétique ont pris une dimension accrue avec le tremblement de terre de 1988 (entre 25,000 et 30,000 morts, un demimillion de sans-abri, un tiers des capacités industrielles de la république détruites). Les Arméniens ont accusé les autorités centrales d'avoir sciemment construit des immeubles en hauteur dans une zone

considérables de la Russie comme instrument d'intégration à l'économie mondiale. Aux yeux des peuples de la périphérie de la Russie, l'exploitation prend donc désormais un visage national beaucoup plus précis qu’avant: il n'est plus «soviétique» mais «russe». Ce qui a fait dire à l'historien Eric Hobsbawm que "Pour la première fois, des populations habituées à l'oppression impartiale de tous (y compris des Grands Russes) par l'autorité centrale avaient des raisons de craindre l'oppression par Moscou dans l'intérêt d'une seule nation ». Hobsbawm, L'Âge des extrêmes. Histoire du Court XXe Siècle, op. cit. p. 638.

34 Un cessez-le-feu est en vigueur depuis 1994.

35 En fait, il s'agirait surtout d’Iraniens turquisés.

36 À la fin de 1990, plus de 160,000 Azerbaïdjanais avaient quitté l'Arménie, et 400,000 Arméniens avaient fait le voyage en sens inverse. Nora Dudwick, «Armenia : paradise regained or lost ?», in Ian Bremmer and Ray Taras (ed.), op. cit., p. 488. 
connue pour son activité sismique et pour avoir fait preuve de lenteur et d'incompétence dans l'organisation des secours. Encore là, la concentration des pouvoirs à Moscou était jugée responsable de l'ampleur du désastre.

L’indépendance de la Biélorussie ne résulte ni de la question environnementale - malgré l'importance qu'elle a eu initialement dans la montée du nationalisme -, ni du nationalisme identitaire, peu répandu dans la population ${ }^{37}$. Comme l'explique Hélène Carrère D’Encausse, "forgée artificiellement, dotée d'une langue qui n'a pas de justification historique, trop proche de la Russie dans son effort pour se démarquer de la Pologne et de la Lituanie, la Biélorussie est, par là même, soumise plus que d'autres à l'influence russe 38 ». Le Parti communiste de Biélorussie (PCB) était dirigé par des conservateurs qui s'opposaient à la transition au capitalisme et qui craignaient le mouvement social déclenché par la perestroïka. En avril 1991, une grève nationale de deux semaines - en réaction à une décision prise par les autorités fédérales - a donné naissance à un mouvement syndical indépendant et renforcé l'idée de souveraineté par la démonstration que le gouvernement soviétique, jugé trop conservateur, n'arrivait plus à satisfaire les besoins des citoyens biélorusses. Craignant un scénario à la Solidarnosc, les dirigeants du PCB ont appuyé le coup d'État conservateur d'août 1991 à Moscou. Toutefois, la victoire de Boris Eltsine et sa décision d'interdire le PCUS sur tout le territoire soviétique a alarmé les dirigeants du PCB, qui décidèrent alors de rompre avec le PCUS dans le but d'éviter le décret visant à le rendre illégal, et de déclarer l'indépendance de la Biélorussie. L’indépendance a donc permis d'éviter à la fois la démocratisation et le passage au capitalisme. Depuis 1994, le pays est dirigé par Aleksandr Loukachenko, réputé plus russophile que les dirigeants russes eux-mêmes (la langue biélorusse n'est parlée que par une minorité) et qui a tenté - sans succès jusqu'à présent - de fusionner son pays à la Russie. Dans la même veine, certains dirigeants d'Asie centrale finiront par avoir recours à l'indépendance pour conserver le pouvoir, en se mettant à l'abri du processus de démocratisation qui avait mené au

37 Au référendum déjà évoqué du 17 mars 1991, 83\% des Biélorusses appuyaient le projet d’Union rénovée proposé par Gorbatchev.

38 Hélène Carrère D’Encausse, L'empire éclaté. La révolte des nations en U.R.S.S., Paris, Flammarion, 1978, p. 272-273. 
renversement des autres dirigeants liés à la période communiste dans plusieurs républiques européennes de l'Union soviétique. À l'inverse, la Kirghizie a déclaré l'indépendance tout juste après la tentative de coup d'État des conservateurs contre Gorbatchev, dans le but d’accélérer la transition à la démocratie et la privatisation.

L'importance des facteurs purement identitaires pour expliquer l'éclatement des fédérations de l'Est reçoit d'ailleurs son démenti le plus sévère dans l'attitude des républiques d'Asie centrale face à la désagrégation de l'URSS. Pourtant, les peuples de cette région se distinguaient foncièrement des populations slaves, tant du point de vue des langues parlées - des dérivés du turc et du persan - que de la religion (l'Islam), de la culture et de la croissance démographique, très élevée. Comme tous les autres peuples de l'URSS, ils se plaignaient du gouvernement central soviétique, qui leur avait notamment imposé la monoculture du coton. En vertu de tout cela, les républiques d'Asie centrale auraient dû figurer parmi les premières à vouloir accéder à l'indépendance.

Tel ne fut pas le cas. Au Kazakhstan, le président Nazarbaïev ne croyait pas en la viabilité d'une transition à l'économie de marché sans la Russie. Il a certes cherché à accroître le contrôle de sa république sur les ressources, notamment minières, mais n'a accepté l’indépendance qu'en étant placé devant le fait accompli : la décision des trois républiques slaves de mettre fin à l'Union. Même après le coup d’État raté d'août 1991, il proposait un plan au Soviet suprême de l'URSS pour former un nouveau gouvernement de l'Union.

En Ouzbékistan, il n'était pas davantage question de sortir de l'Union. Seule la paralysie du gouvernement soviétique après la tentative avortée de coup d’État en août 1991 a incité le gouvernement ouzbek à déclarer l'indépendance, le $1^{\mathrm{er}}$ septembre. Et encore, comme l'explique Gregory Gleason, «les déclarations de souveraineté et d'indépendance de l'Ouzbékistan allaient moins loin que celles des autres républiques soviétiques. Elles étaient conçues pour servir d'instruments dans les négociations avec Moscou plutôt que pour redéfinir le système de gouvernance ouzbek 39 ».

39 Gregory Gleason, «Uzbekistan : the politics of national independence», in Ian Bremmer et Ray Taras (ed.), op. cit., p. 580. 
Le Tadjikistan a été la dernière république à déclarer son indépendance. Elle n’avait pas tellement le choix : l’URSS avait été dissoute. Cela ne signifie évidemment pas que les Tadjiks n’entretenaient aucun grief contre le gouvernement central et contre les Russes, bien au contraire. Néanmoins, le sentiment nationaliste était si faible que même les élites tadjikes le reprochaient à leur peuple : "Même chez plusieurs nationalistes tadjiks instruits des milieux urbains, on voyait les Tadjiks ordinaires, en particulier ceux de la campagne, comme ignorants de leur héritage national et enclins à une forme d'identité locale plutôt que nationale 40 ».

L’URSS a implosé. Toutes ses parties constitutives se sont séparées du gouvernement central, certaines en procédant à des consultations populaires, d'autres sur simple décision du gouvernement. À cet égard, le cas Canada/Québec se révèle encore une fois foncièrement différent. Il ne s'agit pas de dix provinces ou de deux nations (sans compter les autochtones) qui cherchent à se séparer du gouvernement central dans une période de bouleversement politique, économique et social mais d'une seule, dans un contexte de stabilité comparable à celui des autres vieilles démocraties libérales.

L’URSS a éclaté parce que la lutte pour la démocratisation et pour sortir de la crise économique et écologique a suscité un mouvement de masse contre le gouvernement central. Rien de semblable n'existe actuellement au Canada. Par ailleurs, les élites économiques des républiques de l’URSS étaient favorables à la sécession, ce qui n’est pas vraiment le cas au Québec, sauf peut-être - et encore - dans les couches inférieures de la bourgeoisie.

$$
* * *
$$

L'effondrement des régimes en vigueur en URSS et en Europe centrale et orientale a été à la fois cause et conséquence d'une crise

40 Muriel Atkin, «Tadjikistan : reform, reaction, and civil war», in Ian Bremmer et Ray Taras (ed.), op. cit., p. 607. Ce point de vue vaut pour l'ensemble de l'Asie centrale. Voir G. Smith, V. Law, A. Wilson, A. Bohr et E. Allworth, Nation-Building in the Post-Soviet Borderlands: The politics of National Identities, Cambridge (UK), Cambridge University Press, 1998, p. 141. 
multidimensionnelle. En simplifiant, on pourrait presque avancer que ce n'est pas le nationalisme qui est à l'origine du changement qui a mené à l'éclatement des trois fédérations de l'Est. C'est au contraire le changement entrepris dans le contexte de cette crise qui a alimenté le nationalisme.

L'impact du changement politique et social dans les trois fédérations de l'Est a été sans commune mesure avec les promesses qui animent aujourd'hui le mouvement indépendantiste québécois. Là où l'indépendance a résulté d'une large mobilisation, elle a été alimentée par une forte aspiration à s'émanciper de la tutelle d'un État central oppresseur, perçu comme un obstacle à la démocratisation et comme le dernier rempart d'une bureaucratie impuissante à aider le pays à sortir de la crise et à répondre aux aspirations de la société, y compris celles d'une nouvelle classe dominante en devenir.

Au Québec, le mouvement indépendantiste est inféodé à un parti politique qui mise essentiellement sur les aspects identitaires et sur une dénonciation des défauts du régime fédéral canadien au moyen d'une rhétorique technocratique. Mais sur le plan économique, social et politique, ce parti maintient une direction éminemment conservatrice par sa défense de l'ALÉNA, sa volonté d'adapter les programmes sociaux et la fiscalité à la «réalité nord-américaine», ou son refus systématique de démocratiser le système électoral chaque fois qu'il en a l'occasion.

Le processus qui a mené à l'éclatement des fédérations de l'Est enseigne au contraire que l'idée d'indépendance ne pourrait se consolider que dans la mesure où elle serait associée à un changement social et politique majeur. Le «nationalisme civique» prôné par le Parti québécois proposait un Québec souverain fidèle aux canons du néolibéralisme. Ce projet, particulièrement bien incarné par Lucien Bouchard, Bernard Landry et mieux encore par André Boisclair, a abouti à un échec complet. Les hautes instances actuelles du PQ, dirigé par Pauline Marois, misent plutôt sur l'identité. Or, à moins de subir une campagne acharnée d'insultes largement publicisées en provenance du Canada anglais, on voit mal comment, si ce qui s'est passé dans les fédérations de l'Est pouvait servir d'indice, une telle stratégie pourrait mobiliser la population en faveur de l'indépendance. Le mouvement indépendantiste québécois a pris son envol dans un contexte où il incarnait le changement sur le plan social, politique, économique et 
culturel. Même en 1995, la remontée des appuis à l'option souverainiste résultait en grande partie de la mobilisation des centrales syndicales, des groupes populaires, du mouvement coopératif, des artistes, des étudiants ${ }^{41}$. Les attentes de ces segments de la population advenant une victoire du «Oui» allaient certainement au-delà des intentions des dirigeants de leur propre camp, Jacques Parizeau et Lucien Bouchard en tête, comme en ont fait foi la grande majorité des rapports qui ont été déposés à la Commission nationale sur l'avenir du Québec. On y revendiquait l'élection d'une assemblée constituante, un changement de système électoral et de mode de représentation, des politiques sociales et culturelles, de développement régional, etc. La gouvernance provinciale exercée par les gouvernements péquistes après 1995 a détruit beaucoup d'illusions à cet égard. L'option souverainiste a cessé, dans l'esprit de plusieurs, d'incarner ces aspirations. Dans les fédérations de l'Est, les batailles menées contre l'État central ont certes été l'œuvre d'un amalgame d'intérêts parfois contradictoires et de motivations de tous ordres, parfois peu défendables. Mais elles avaient toutes pour but de changer la vie quotidienne, de transformer les rapports sociaux. C'est pourquoi il est permis de croire que la promesse d'un Québec (indépendant) plus démocratique et égalitaire que le Canada aura plus de chances de remporter la bataille que celle du statu quo dans un Québec souverain.

Fin du texte

41 Le regroupement de la plupart des organisations patronales dans le camp du «Non» donnait d'ailleurs à la campagne référendaire la forme apparente d'une lutte de classes. 